\title{
Le réseau énergétique en projet
}

\section{Fanny Lopez}

\section{(2) OpenEdition \\ Journals}

Édition électronique

URL : http://journals.openedition.org/crau/493

DOI : $10.4000 /$ crau.493

ISSN : 2547-5746

\section{Éditeur}

Éditions du patrimoine

\section{Édition imprimée}

Date de publication : 1 septembre 2013

Pagination : 25-36

ISBN : 978-2-7577-0109-6

ISSN : $1296-4077$

Référence électronique

Fanny Lopez, "Le réseau énergétique en projet », Les Cahiers de la recherche architecturale et urbaine [En ligne], 28 | 2013, mis en ligne le 12 septembre 2017, consulté le 03 mai 2019. URL : http:// journals.openedition.org/crau/493 ; DOI : 10.4000/crau.493 
L'actuelle multiplication des propositions architecturales et urbaines en faveur de l'autonomie énergétique remet en cause le modèle historique des grands réseaux pensé pour la consommation de masse et la croissance. Les réseaux de services et d'énergie agitent pourtant le projet architectural et urbain depuis un bon siècle, interrogeant la hiérarchie du degré de connectivité et la question de l'échelle, de la petite maison au territoire autosuffisant. Connexion vs déconnexion, macro vs micro, l'histoire des services s'envisage comme une bataille : de courant, d'échelle, de modèle technique, économique et de gouvernance, auxquels les ingénieurs et les architectes n'ont cessé de prendre part. Cet article propose d'interroger I'histoire de l'architecture et de l'urbanisme pour saisir la façon dont la distribution énergétique s'est constituée comme support de projets, dans la réalité comme dans la fiction.

\section{Le réseau énergétique en projet}

FANNY LOPEZ

Le $x x^{e}$ siècle occidental a conjugué l'énergie sur le mode de la centralisation, de l'expansion et de la connexion, vouant à sa puissance le culte de la grandeur. L'immense toile de conduites, de fils et de câbles, et les gigantesques centrales hydro-électriques ou nucléaires, ont définitivement transformé le paysage et bouleversé les processus d'urbanisation. Pourtant, la théorie de l'architecture et de l'urbanisme est relativement discrète sur son rapport aux réseaux de services (eau, assainissement, énergie). À partir du milieu des années 1960, l'ingénierie devient l'élément essentiel du rapport entre l'architecture et l'environnement, mis en avant par James Marston Fitch, Leo Marx', et plus radicalement encore par Reyner Banham ${ }^{2}$, mais la question des réseaux territoriaux n'est que peu abordée. Il faut alors se tourner du côté des historiens des techniques qui ont fait de l'extension des connexions le phénomène majeur de la modernité. Le branchement à l'espace réseau a notamment été théorisé par Gabriel Dupuy dans L'urbanisme des réseaux, théorie et méthodes. La connexion définit la circulation des personnes,

1. James Marston Fitch, American Buildings: The Historical Forces that Shape it (vol. 1), The Environnemental Forces that Shapes it (vol. 2), New York, Schocken Books, [1947] 1971 Leo Marx, The Machine in the Garden : Technology and the Pastoral Ideal in America, New York, Oxford University Press, 1964
2. Reyner Banham, L'architecture de l'environnement bien tempéré [The Architecture of the Well-Tempered Environment, Londres, The Architectural Press, 1969], Orléans, HYX, 2011. Voir notamment l'introduction de Luc Baboulet qui replace l'ouvrage dans les débats de l'époque. 
des biens, des fluides, des informations; c'est la condition de l'activité économique, de l'urbanité ${ }^{3}$, ce sont les réseaux, tous les réseaux. Aujourd'hui, la problématique environnementale et l'accélération des recherches sur la métropole post-carbone nous invite à l'introspection disciplinaire de l'héritage de cet urbanisme des réseaux, faisant ressurgir des problèmes qui semblaient résolus depuis longtemps.

La multiplication des appels en faveur de la transition énergétique questionne la durabilité du modèles des grands réseaux de services construits pour la consommation de masse et la croissance, interrogeant les travaux relatifs à ses alternatives et à sa diversité infrastructurelle.

Cet article propose de sonder l'histoire de l'architecture et de l'urbanisme pour voir la façon dont la distribution énergétique n'a cessé d'apparaître comme support de projet, de grande ou de petite échelle, dans la réalité comme dans la fiction.

\section{Assurer les branchements}

\section{Du pragmatisme à l'utopie}

Dès la fin du XIX siècle, la question de la rationalisation des services urbains et de la connexion s'intensifie. Ildefonso Cerdà, est dans la théorie urbaine un de ceux qui consacrent aux fluides et aux flux le plus de pages en termes de technique, de gestion, de gouvernance. Dans sa célèbre Théorie générale de l'urbanisation, il appelle à prendre toute conscience des tuyaux par lesquels coulent les fluides de la ville. "On dirait à première vue que ces différents appareils forment le système veineux d'un être mystérieux aux dimensions colossales. [...] cet ensemble de tubes ne constitue rien d'autre qu'un système d'appareil qui entretient le fonctionnement de la vie urbaine ${ }^{4}$. " II y a une réponse pragmatique et technique aux problématiques sanitaires : réorganisation du sous-sol, implantation, rassemblement et hiérarchisation des conduites. Pour Cerdà, une fois le réseau en place, le développement urbain est facilité : si de nouvelles constructions voient le jour, « il suffit d'expédier le tout sous terre en mettant en communication les tuyaux ${ }^{5}$ ".

Les réseaux de services sont un élément structurant de la planification urbaine et territoriale et le plan présenté pour Barcelone impulse et accompagne un mouvement qui va toucher, dans les décennies suivantes, un grand nombre de cités d'Europe.

Si dans les premiers temps de sa structuration, le réseau est indissociable de l'architecture, il est généralement solutionné comme une donnée technique parallèle et organisationnelle indépendante du projet architectural et urbain. Le réseau relie, mais c'est une conception urbaine non extensive. Différemment, dans la cité linéaire de Arturo Soria y Matà ${ }^{6}$ et plus manifestement encore chez les désurbanistes russes, il est moteur du projet et la question qui se pose est celle du grand territoire et des ressources disponibles. L'énergie s'apprivoise sur un mode expansionniste.

Au début des années 1920, I'urbanisation et, plus largement, l'aménagement du territoire soviétiques sont soumis à l'électrification du pays avec le plan Goelro' qui prévoit sur une quinzaine d'années la construction d'une trentaine de centrales électriques régionales pour assurer de façon concomitante le développe-
3. Gabriel Dupuy, L'urbanisme des réseaux : théorie et méthodes, Paris, Armand Colin, 1991. L'auteur réhabilite dans ce texte les théories urbanistiques favorables aux réseaux en revenant sur la vision globale de ceux qu'il considère comme ses trois pionniers : Ildefonso Cerdà, Frank Lloyd Wright et Maurice-François Rouge. II insiste sur les dimensions typologique, cinétique et sur l'adaptabilité du système viaire chez Wright et Cerdà. L'approche est moins sectorielle chez Rouge, qui appréhende les réseaux comme un phénomène structurel et territorial, voire infini.

4. Ildefonso Cerdà, Teoría general de la urbanización, y aplicación de sus principios y doctrinas a la reforma y ensanche de Barcelona, Madrid, Imprenta Española, 2 vol., 1867. Trad. fr., La théorie générale de I'urbanisation, présentée et adaptée par Antonio Lopez de Aberasturi, Paris, Seuil, 1979, p. 94.
5. Id., p. 142

6. Conçu en 1882, le principe urbain de la cité linéaire d'Arturo Soria y Mata rassemble dans l'axe de la rue principale les circulations et l'ensemble des services: téléphone, chauffage, gaz, eau, électricité.

7. Goelro, abréviation russe signifiant "Commission d'État pour l'Électrification de la Russie ». 
ment industriel et l'établissement de villes nouvelles ${ }^{8}$. Les désurbanistes défendent l'idée de la dispersion de l'industrie et de l'habitat sur tout le territoire afin d'assurer au mieux la fusion entre ville et campagne. Ils proposent des axes linéaires de transport et de services autour desquels pourraient se développer des communautés industrielles et agricoles. La puissance électrique joue un rôle de premier plan. Les propositions d'Ivan Ilich Léonidov ou de Nikolaï Milioutine témoignent des espoirs qu'elles suscitent, avec comme point commun un traitement par bandes linéaires organisées autour d'un axe infrastructurel proche de la conception de Soria y Matà. La centralisation électrique est la condition du déploiement territorial. Pour Mikhail Okhitovitch, "le réseau supplantera le centre. Ce ne sont pas tant les centres d'énergie eux-mêmes qui sont importants que l'interconnexion des centres de moindre importance en un seul et unique réseau d'énergie. Le passage de l'utilisation des centres d'énergie à celle d'un grand réseau bouleverse totalement le problème de l'épuisement des sources d'énergie dans le monde?."

Paradoxalement, la centralisation de l'énergie électrique est la condition de la dispersion. Avec les désurbanistes, c'est l'une des premières fois qu'une discipline liée à l'aménagement de l'espace revendique avec autant de détermination l'électricité comme support explicite du projet architectural et urbain. Le réseau électrique structure le territoire et devient la matrice projectuelle. La connexion et les systèmes énergétiques de grande échelle ont, à cette époque, une valeur utopique.

8. Anatole Kopp, Changer la vie, changer la ville. De la vie nouvelle aux problèmes urbains URSS 1917-1932, Paris, Union générale d'éditions, 1975, p. 282-290.

9. Mikhail Okhitovitch, "Remarque sur la théorie du peuplement », SA (Sovremmennaia Arkhitektura soit " architecture contemporaine »), $\mathrm{n}^{\circ}$ 1-2, 1930.
Il faut avoir en tête qu'en Europe comme aux ÉtatsUnis, l'interconnexion des réseaux électriques est un projet techniquement et économiquement difficile avant le début du xx siècle. Influencé par la fascination des Russes pour l'électricité, c'est Herman Sörgel, qui va pousser à la démesure le projet infrastructurel de la modernité. En 1928, avec Atlantropa, I'architecte allemand allait jusqu'à se demander si l'on pourrait fabriquer suffisamment de béton pour architecturer le barrage hydro-électrique du détroit de Gibraltar, composé d'une écluse surmontée d'un bâtiment de $400 \mathrm{~m}$ et d'un belvédère. Atlantropa apparaît comme le projet paroxysmique d'une génération vouant à la puissance de l'énergie le culte de la grandeur. Désireux d'amalgamer I'Afrique coloniale, voire le continent entier, à une Europe unie, la technique et l'ingénierie sont le socle du projet qui visait - entre autres - à assécher partiellement la Méditerranée pour libérer des terres arables, transformer le Congo en lac artificiel, construire un réseau de centrales hydro-électriques et relier Berlin, Rome et la ville du Cap par voie ferrée.

Ce projet colonialiste de super continent ambitionnait de répondre à la menace de l'épuisement des ressources en charbon et en pétrole. Atlantropa marque l'apogée d'une nouvelle génération d'utopies techniques qui « coïncide avec l'épanouissement de la deuxième révolution industrielle : outre l'expansion de la chimie, celle-ci se caractérise avant tout par l'électrification massique des pays industrialisés $[\ldots]^{10} "$.

Le $x x^{e}$ siècle a entériné le modèle industriel des grands réseaux et les architectes vont se l'approprier comme un objet support de projet. II y a toutefois de longs silences

10. Wolfgang Voigt, "Le plus grandiose projet des temps modernes », dans Hubert Damisch et Jean-Louis Cohen (dir.), Américanisme et modernité : l'idéal américain dans l'architecture, Paris, EHESS/ Flammarion, 1996, p. 378. 


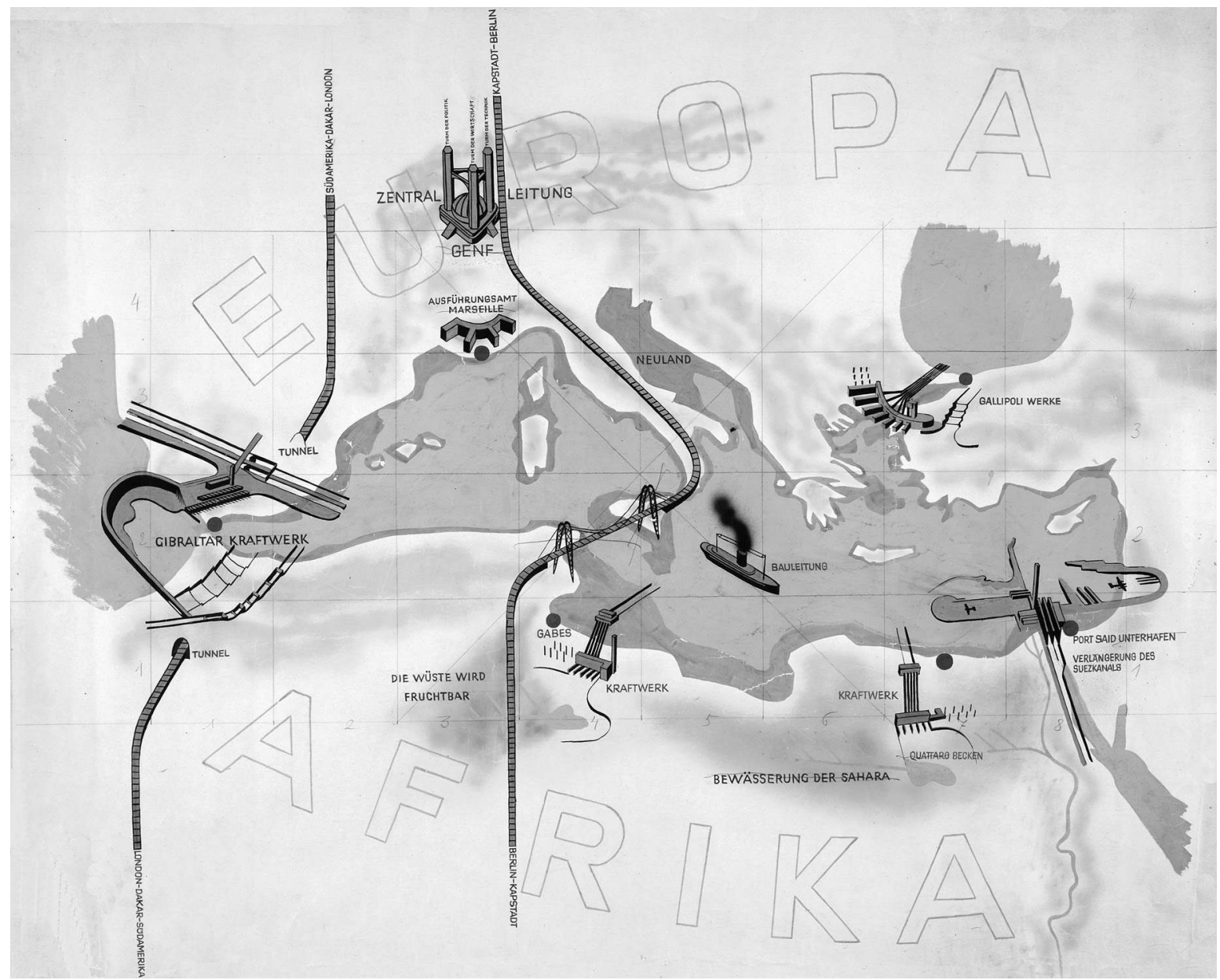

Herman Sörgel, projet

"Atlantropa », 1928.

CWolfgang Voigt, Atlantropa,

Weltbauen am Mittelmeer, Ein

Architektentraum der Moderne,

Hambourg, Dolling und Galitz

Verlag, 1998. 
sur la chose énergétique dans la théorie architecturale et urbaine. N'est-ce pas Richard Buckminster Fuller qui déclara à propos des architectes du Mouvement moderne : "Ils n'ont jamais enquêté sur les problèmes énergétiques ou sanitaires. Ils se sont installés sur les égouts comme des poules sur des œufs de verre ${ }^{11}$ ". Si le réseau est pour nombre d'architectes un acquis, il est l'affaire des ingénieurs et des aménageurs. C'est un à-côté de modernité, les architectes s'en préoccupent

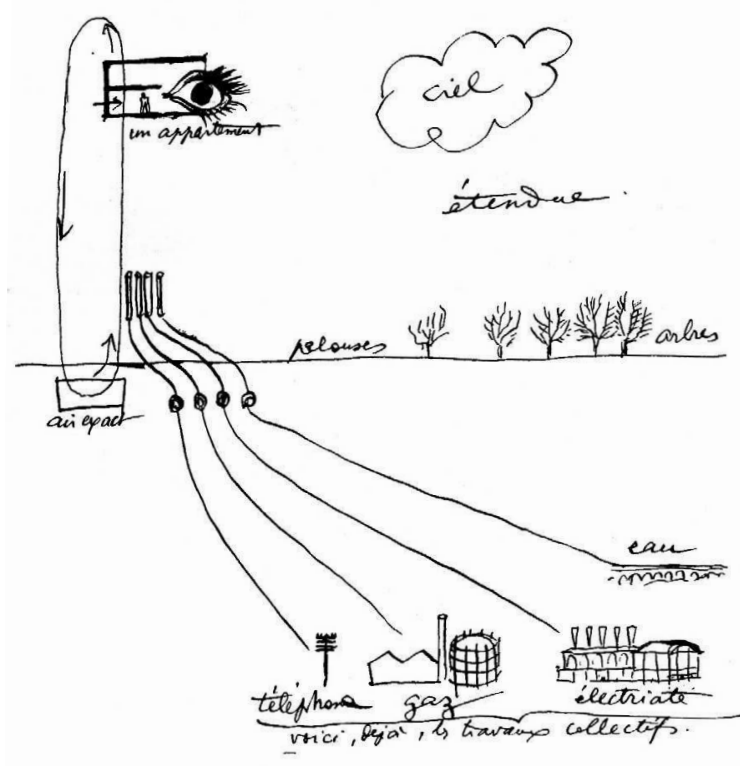

peu, ses représentations reste rares et il n'est jamais remis en doute. De fait, la modernité est branchée. Le Corbusier utilise le terme de "terrain artificiel »pour qualifier ce nouvel état du territoire, artificialisé, maillé par ces «formidables » réseaux en tous genres. Ses unités d'habitations, viennent se brancher à des infrastructures lointaines, qu'il représente presque caricaturalement : les arcs de l'aqueduc pour l'eau, les sheds et les cheminées fumantes pour le gaz et l'électricité.

$\mathrm{Ce}$ sont des machines productives industrielles mises à distance de son architecture. Or aujourd'hui, les préoccupations environnementales s'appliquent à réduire cette distance, faisant fusionner dans une hybridité revendiquée l'architecture et l'infrastructure, soutenue par l'idée de la réduction des temps de parcours et de la relocalisation des sources énergétiques.

Les problématiques techniques des dix-neuviémistes résolues, le réseau est devenu un instrument territorial inspirateur du projet, dont la grande échelle semble attester du choix de la connexion comme modèle dominant. Mais, il ne faut pas oublier qu'au moment même où le rêve de la connexion se réalise, transformant en un laps de temps très court chaque citadin en abonné de la modernité, l'idée de la déconnexion apparaît.

\section{Disconnection is beautiful}

L'annonce d'une catastrophe climatique annoncée nous a donné depuis plus d'une décennie quelques signes tangibles du retour du «petit ». On se souvient alors avec nostalgie de cette année 1882 où la première petite centrale électrique d'Edison se mit à tourner au creux du tissu urbain de Manhattan, alimentant
Le Corbusier,

" Le terrain

artificiel ", La

ville radieuse,

1933.

(C) FLC/ADAGP, 2012
11. Fuller cité par Reyner Banham, Architectural Design $n^{\circ} 31$, juillet, 1961, p. 295. 
quelques 80 appartements et bureaux. L'énergie était là, dans la ville, visible et mesurable, presque tangible.

Ce même Edison présentait en 1912 une maison électriquement indépendante des réseaux : "la résidence suburbaine du xxe siècle ». Elle offrira, écrit-il, « une énergie bon marché et autonome à tout pavillon de banlieue ainsi qu'aux maisons en zone rurale, situées en dehors des lignes de distribution de la ville ${ }^{12}$. "Avec ce projet, Edison répond à une véritable demande. Dans les centres urbains, le raccordement ne concerne que les plus privilégiés et la production " autonome " ou «à la cave » d'électricité est l'apanage de quelques grandes entreprises qui ont les moyens de gérer un tel dispositif. Pour les autres, l'accès à ce service se fait attendre. Edison estima le coût du système à 500 \$. La première maison opérationnelle est celle de

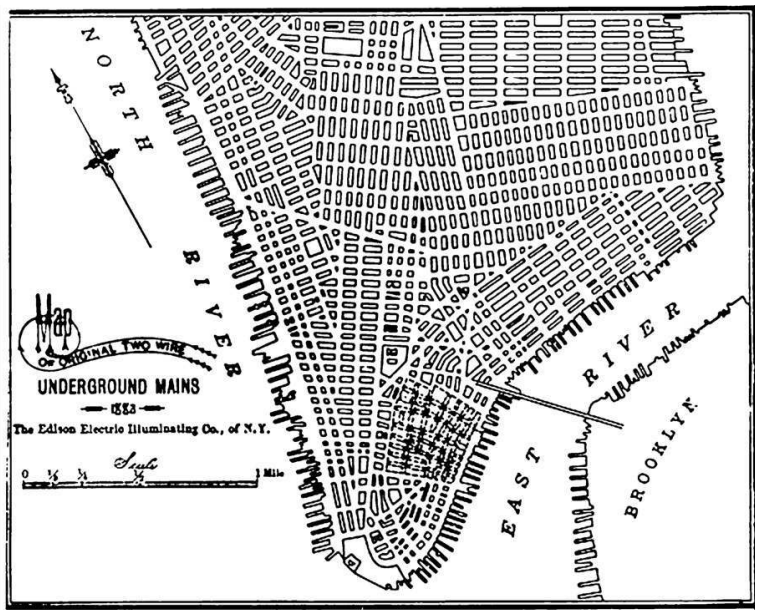

son concepteur à Llewellyn Park dans le New Jersey. À une époque où la critique des monopoles bat son plein, la presse applaudit : " Tout le monde peut maintenant avoir une petite usine électrique dans sa propre cave à un coût relativement modeste qui offre lumière et chaleur et facilite les tâches domestiques. Chacun peut être indépendant et pour tous les temps des grandes entreprises électriques ». Mais face à la férocité en affaires de l'autre géant de l'électricité, Georges Westinghouse, Edison a dû abandonner ses recherches sur le courant continu, les microréseaux et l'autonomie pour rester dans la course et fonder son empire : la General Electric. Le fondateur de l'un des plus importants conglomérats industriels du monde s'est intéressé à l'autonomie énergétique, il y a un siècle...

Avant les années 1920, aux États-Unis comme en Europe, l'hétérogénéité énergétique et la concurrence entrepreneuriale ont prévalu jusqu'à l'harmonisation, sous la coupe du service public, des distributions, des voltages et des compteurs au nom de la sécurité et de l'égalité énergétique. C'est la naissance des grands réseaux et des services pour tous, mais le projet de la déconnexion ne va cesser de bousculer cet ordre énergétique. Richard Buckminster Fuller se joue de leur disparition dès 1929 en supprimant, dans sa célèbre Dymaxion House, toute nécessité de branchements. Si la théorie urbaine a consacré peu de pages au réseau énergétique, on peut alors retraverser l'histoire de I'architecture et de l'urbanisme et établir un catalogue du micro, en reconnaissant à un Frank Lloyd Wright son engagement dans la défense de la décentralisation et

Edison, Retadl SstriedtaStattidarvel, The 


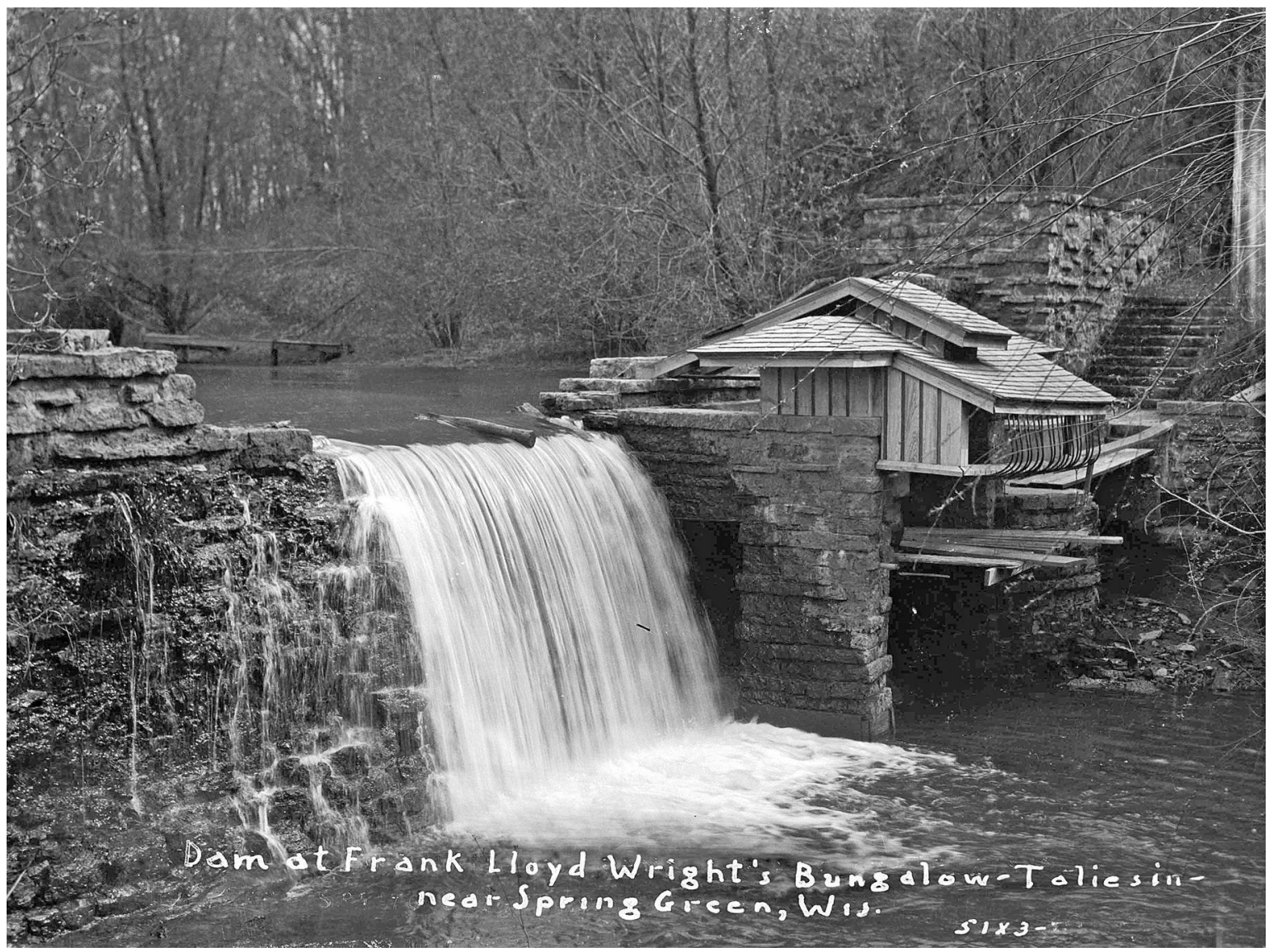

Frank Lloyd Wright, la petite centrale hydroélectrique de Taliesin, 1910.

CWisconsin Historical

Society. Image ID 60721

(www.wisconsinhistory.

org/whi/fullRecord.

asp ?id=60721). 
ses mises en application à Taliesin. C'est toute l'habileté de sa tour éolienne ou de sa centrale hydro-électrique qui préfigurait déjà l'ambition de Broadacre City...

Wright défendra clairement tout au long de sa carrière, et encore plus lors de la crise de 1929, la diversification des sources (hydraulique, fossile et éolienne) et la construction de barrages ou de forages de petites échelles à l'image des communautés agro-industrielles qui les exploiteraient. Ainsi disparaîtraient les lourdes infrastructures de la cité industrielle pour un paysage biotechnologique réconcilié.

Mais c'est probablement la fin des années 1960 qui marque une nouvelle étape dans la critique et dans la déconstruction des grands systèmes énergétiques. La petite échelle va s'envisager comme le lieu privilégié de l'expression de la démocratie. Avec la remise en cause du discours moderniste, c'est aussi la crise de l'aménagement; le retour de l'individu est marqué par un grand désir de participation et d'intervention. La publication d'Ernst Friedrich Schumacher, Small is beautiful ${ }^{13}$ deviendra le slogan d'une génération qu'accompagneront les publications d'un Dennis Meadows ouvrant à la question des ressources et de l'énergie un champ de questionnements. Dans le sillage du choc pétrolier de 1973, le mouvement de I'autonomie énergétique se structure. Dépassant les limites de la contre-culture américaine où il a souvent était cantonné, le mouvement se propage géographiquement, remontant des filières plus institutionnelles et poussant ses propres limites, de l'unité domestique à la ville territoire. La maison autonome d'Alexandre Pike ou les projections d'Alexandroff pour la cité auto-énergétique apparaissent comme autant de parades mécaniques, se substituant à la grille énergétique centenaire ${ }^{14}$.

Du passé, faisons table rase : dans une négation de l'existant, voici disparu le réseau et ses infrastructures. L'autonomie énergétique n'est plus un tabou aux relents sécessionnistes : se déconnecter, ce n'est plus se couper du monde, mais juste un modèle énergétique. Le lointain est devenu proche, l'énergie est juste là, dans certain cas elle est l'architecture.

\section{La diversité infrastructurelle face au macro-système technique}

Au début des années 1980, les protagonistes de l'autonomie énergétique mettent en garde ceux qui pensent détenir avec les énergies renouvelables une technologie inajustable à ce que les socio-anthropologues appellent

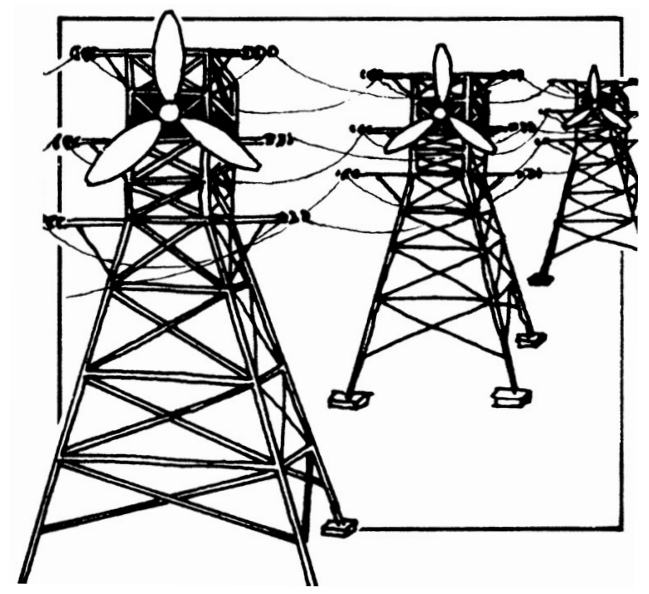

13. Ernst Friedrich Schumacher, Small Is Beautiful: A Study Of Economics As If People Mattered, 1978. Trad. fr. Small Is Beautiful. Une société à la mesure de I'homme, Paris, Seuil coll. «Points», 1978
14. Fanny Lopez, Le rêve d'une déconnexion, Paris, Éditions de la Villette, à paraître en 2013. Voir également

"Vers le grand débranchement? », dans

Dominique Rouillard (dir.), L'Infraville. Futurs

des infrastructures, Paris, Archibooks,

2011, p. 117-127 et «L'utopie énergétique

d'Alexander Pike », dans Éric Alonzo et

Sébastien Marot (dir.), Marnes, documents

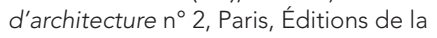

Villette, 2011, p. 133-167.
Éoliennes en réseau. (c) Peter Harper, Godfren Boyle, Radical Technology, Londres, Wildwood House, 1976. 
le macro-système technique ${ }^{15}$. Cette entité techniqueadministrative construite et qui n'a cessé de se renforcer depuis la fin de XIX siècle. Le macro-système technique, $c^{\prime}$ est le réseau surtout électrique et ses infrastructures dans leur large organisation structurelle. Pourtant, les années 1980 ont engagé une soumission des systèmes énergétiques alternatifs aux réseaux.

Les énergies renouvelables présentent souvent les mêmes caractéristiques de centralisation institutionnelle et physique que le nucléaire et les énergies fossiles. Les outils technocratiques et bureaucratiques se sont réorientés vers la protection de l'environnement, sans pour autant modifier les règles de dépendance vis-à-vis du système centralisé. Au début des années 1990, la mise en œuvre des Agendas 21, puis le lancement des Conférences européennes des villes durables activent une mobilisation à tous les niveaux institutionnels, donnant un tour plus concret à l'action environnementale urbaine. Cette impulsion s'est traduite notamment par la réalisation d'éco-quartiers, d'abord dans les villes du nord de l'Europe. Ce sont les désormais célèbres : Bedzed (Beddington Zero Energy Development) au sud de Londres, Harmmarby ou Björkhagen à Stockholm. De nombreux exemples dans des temporalités et des contextes différents ont su engager une gestion relocalisée de la production et du traitement des fluides élémentaires dans un objectif revendiqué d'autonomie, expérimenté et souvent atteint.

Si la reconstruction de micro-réseaux a déjà prouvé son efficacité technique à l'échelle d'une maison, d'un bâtiment, d'un quartier, d'une ville, voire d'un territoire ou d'une île, le consensus énergétique et les défenseurs du modèle historique préfèrent les notions de système hybride, de support partiel, d'allègement. En France, un ensemble d'institutions et de circulaires tente d'encadrer ces pratiques en devenir, mais la réglementation énergétique a encore l'air d'un habit de confection mal ajusté. L'évolution des normes passant de HOE (haute qualité environnementale) à BEPOS (bâtiment à énergie positive) et l'engagement du Grenelle sur les éco-quartiers et éco-cités marquent une évolution, même si la grande majorité des bâtiments est raccordée au réseau électrique centralisé. Plutôt que l'autonomie d'unités ou d'ensembles d'habitations, la majorité des partenariats européens publics-privés vantent actuellement la durabilité de bâtiments interconnectés au réseau électrique centralisé et régis par le principe des équilibres énergétiques en temps réel, dont la smart grid apparaît comme un nouvel idéal d'optimisation.

Aujourd'hui cette intégration est en passe d'être la norme et toute la question du futur énergétique semble se résumer à l'interconnexion : comment brancher ces micro-réseaux aux macros, comment faire du macro avec les énergies renouvelables ? Pour l'électricité, les micro-réseaux sont invités, voire contraints, de se raccrocher à la super-grid. " Micro-grid», "smart grid " et "super-grid », sont les maîtres mots d'un nouvel ordre énergétique complémentaire.

Le système réticulaire de grande échelle continu d'être le support de projections économiques et spatiales, internationales ou intercontinentales, à l'exemple de Desertec qui prévoit, à partir des ressources renouvelables du désert nord-africain, la création d'un réseau électrique qui alimenterait l'Afrique

15. Alain Gras, Les macro-systèmes techniques, Paris, PUF, Que sais-je, 1997 ; "Les réseaux, les machines et la mégamachine : sur l'origine des systèmes techniques contemporains ", dans Pierre Musso (dir.), Réseaux et société, Paris, PUF, 2003. 
du Nord, l'Europe et le Moyen-Orient. La prévision de I'OMA pour Roadmap 2050 est similaire : Eneropa est un réseau d'énergie renouvelable interconnecté à l'échelle européenne ${ }^{16}$. La carte de l'Europe est ainsi renommée en fonction des potentialités énergétiques de ses régions.

L'utilisation du modèle de la carte du métro londonien témoigne de l'importance de ces interconnexions pour un réseau de si grande ampleur. Comme ce fut le cas tout au long de I'histoire, connexion et déconnexion coexistent. Si la prospective énergétique se poursuit dans la tradition historique de la connexion de grande échelle, les potentialités de systèmes décentralisés, voire autonomes, n'en continuent pas moins d'être sollicités par quelques personnalités du monde de l'aménagement.

Depuis le début des années 2000, on observe une réactualisation de la problématique de l'autonomie dans le champ de l'architecture et de l'urbanisme. Une recrudescence de projets et de publications attestant de l'ampleur de ce phénomène. Dans la ligne de I'utopie technique, il y a un renouveau de la tradition méga-structurale qui fait de l'énergie l'objet même du projet. Des architectes imaginent des monuments énergétiques futuristes allant des immeubles de grande hauteur à des solides métropolitains auto-énergétiques. La bigness a gagné l'énergie, à l'exemple des résultats du concours 2012 " eVolo Skyscraper Competition ", I'une des récompenses les plus prestigieuses pour l'architecture de gratte-ciel, ou encore de ceux issus de la troisième "Compétition d'Architecture Avancée » organisée par l'Institut d'Architecture
Avancée de Catalogne sur la ville autosuffisante ${ }^{17}$. Le projet énergétique se construit comme une icône.

Une autre orientation s'inscrit dans la tradition architecturale et technique plus institutionnelle, dite high-tech. Des équipes d'architectes et d'ingénieurs mettent au point des objets aussi performants que coûteux. Ce sont des objets solitaires dans le sens où ils réinventent à leur échelle un système de services intégrés. L'autonomie est notamment justifiée dans des environnements vierges d'infrastructures énergétiques. C'est le cas du bâtiment édifié sur les sommets montagneux du Valais suisse par l'École polytechnique fédérale de Zurich. Ce refuge de cing étages pouvant accueillir 120 personnes a été réalisé en 2009 pour le Club alpin suisse, et il assure la production de ses propres besoins en eau et en énergie. À une échelle différente, la ville nouvelle de Masdar à Abu Dhabi planifiée par Norman Foster dans le désert des Émirats arabes unis illustre la création de nouvelles matrices énergétiques pour les énergies renouvelables. Dans des zones déjà desservies par le réseau, le parti pris de l'autonomie est plus difficile à défendre. Le bras de fer engagé par le siège social d'Abalone - livré en 2006 par C\&Cie J. L. Cousin à quelques kilomètres de Nantes - pour une déconnexion totale du réseau ERDF témoigne de la difficile acceptation d'une autonomie totale, perçue comme une véritable défiance énergétique faite au service public existant, cet élément structurant de solidarité urbaine ${ }^{18}$. Mais à la création ex nihilo de nouveaux réseaux énergétiques, vient se poser la question de l'existant, de sa réhabilitation et de sa continuité.
16. En 2009, la Fondation européenne pour le climat (European Climate Foundation) a lancé une étude sur l'énergie intitulée "Feuille de Route 2050 » (Roadmap 2050) afin de susciter des proposition en vue d'une réduction drastique des émissions de $\mathrm{CO}_{2}$ à l'horizon de 2050. Rem Koolhaas et I'OMA (Office for Metropolitan Architecture) faisaient partie des consultants.
17. IAAC, Self-Sufficient City. Envisionning the Habitat of the Future, Barcelone, Actar, 2010

18. G. Dupuy, L'urbanisme des réseaux: théories et méthodes, op. cit. note 3 . 


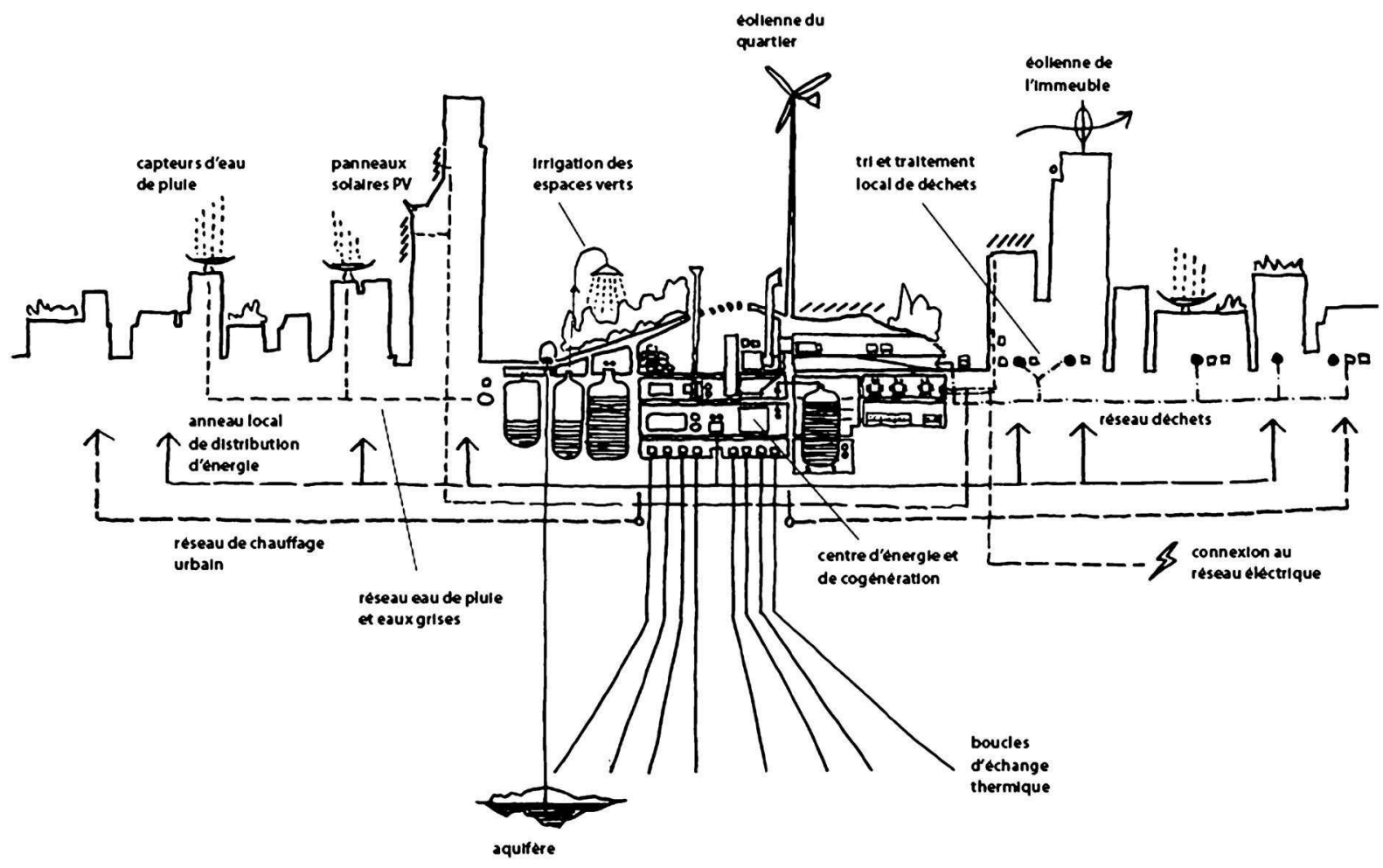

Richard Rogers, pile énergétique, 2008. CGPAP 


\section{Archéologie énergétique pour un territoire renouvelé}

Le territoire existant recèle des architectures industrielles partiellement désuètes ou en attente de reconversion. Il y a une nouvelle lecture du territoire qui se construit, elle passe par une véritable archéologie infrastructurelle. Aux enclaves énergétiques monofonctionnelles (la centrale hydro ou thermo-électrique) se substituent des polarités énergétiques polyfonctionnelles.

En France, la Consultation internationale « le grand pari de l'agglomération parisienne » lancée en 2008 a donné à la question énergétique les signes d'une reprise en main et d'un certain pragmatisme. L'équipe de Richard Rogers a ainsi proposé par exemple de restructurer la ville compacte autour d'armatures métropolitaines multifonctionnelles, certaines comportant des piles énergétiques.

Ce sont des centres techniques de production d'énergie et de ressources incrustés dans le tissu urbain ou périphérique. L'incinération des déchets peut produire de la chaleur et générer de l'électricité, les déchets biomasse sont utilisés pour la méthanisation. Ces pôles énergétiques composent des micro-réseaux, partiellement branchés sur les grands. Dans une hybridité revendiquée, architecture et infrastructure reconstituent un paysage urbain post-carbone. L'équipe Lin menée par Finn Geipel et le Studio 9, dirigé par le duo Bernardo Secchi et Paola Viganò, ont également proposé de resegmenter et recycler la matrice infrastructurelle pour la rendre flexible et créer de nouvelles interactions.

Dans le cadre du programme de recherche Ignis Mutat Res, Penser l'architecture, la ville et les paysages au prisme de l'énergie ${ }^{19}$, l'équipe Viganò poursuit ses travaux sur les ressources, en se concentrant sur le recyclage de l'énergie grise et l'autosuffisance. Toujours dans le cadre de cette consultation, l'équipe de Philippe Potié, Djamel Klouche et Florian Hertweck s'est concentrée sur les potentialités énergétiques des régions transfrontalières. Le projet urbain et territorial se restructure autour de nouvelles polarités énergéticoindustrielles. Catalogue des ressources, cartographie énergétique et topographie réticulaire sont les outils nécessaires à une nouvelle radiographie du territoire.

Mais la question qui se pose - pour l'électricité de façon toujours aussi manifeste - est celle de l'échelle des réseaux. Les termes réseau primaire, secondaire ou d'appoint témoignent d'un choix hiérarchique et du degré de connectivité. À quelle échelle pense-t-on I'autonomie (et donc le réseau) : à celle d'un bâtiment, d'un îlot, d'un territoire et jusqu'où interconnecter ces différents ensembles? Connexion vs déconnexion, macro vs micro, l'histoire des services s'envisage aussi comme une bataille : de courant, d'échelle, de modèle technique et de gouvernance, auxquels les ingénieurs mais aussi les architectes n'ont pas fini de prendre part.

19. Programme interdisciplinaire de recherche Ignis Mutat Res «Penser l'architecture, la ville et les paysages au prisme de l'énergie ", ministère de la Culture et de la communication, ministère de l'Écologie, du développement durable, des transports et du logement, Atelier international du Grand Paris, 2011-2014. 